\title{
Interessenförderung im Unterricht zum Lernbereich Globale Entwicklung
}

\begin{abstract}
Zusammenfassung
In diesem Beitrag wird die Frage nach Interessenförderung im Unterricht zu globalen Themen bearbeitet. Zunächst legt die Autorin dar, dass die Förderung von Interesse im Kontext des Diskurses um Globales Lernen eine relevante Zieldimension ist, die aber nur bedingt expliziert und dabei theoretisch sowie konzeptionell reflektiert wird. Hieran anschließend werden vor dem Hintergrund zentraler Theorien zur Interessenentwicklung aus der Pädagogischen Psychologie theoretische wie empirische Erkenntnisse im Feld Globalen Lernens betrachtet und diskutiert.
\end{abstract}

Schlüsselworte: Globales Lernen, Interessenentwicklung, Unterricht im Lernbereich Globale Entwicklung

\begin{abstract}
This article deals with the role of interest development in lessons on global issues. The author notes that although the promotion of interest is a central objective, it is only partially explicated and thus theoretically and conceptually reflected in the discourse on global learning. Against the background of central theories of interest development, theoretical as well as empirical findings in the field of global learning are considered.
\end{abstract}

Keywords: Global learning, interest development, teaching global development

\section{Interesse und Globales Lernen - Defizite auf theoretischer und konzeptioneller Ebene}

Wenn in schulischem Unterricht über Globalisierung gesprochen wird, werden im Sinne des Globalen Lernens auch ökologische und soziale Problemlagen thematisiert. Dabei besteht wohl Konsens dahingehend, dass es Zielperspektive eines solchen Unterrichts ist, dass Jugendliche für Fragen des globalgesellschaftlichen Zusammenlebens sensibilisiert werden, lernen über ihr eigenes Handeln und dessen Auswirkungen nachzudenken und sich zukünftig auch selbstständig mit Missständen in der Welt auseinandersetzen. Implizit vorausgesetzt wird dabei die Zielperspektive, das Interesse der Schülerinnen und Schüler für globale Heraus- forderungen zu wecken, um sie zu einer andauernden - d.h. einer auch außerhalb der Schule relevanten und in ihrem späteren Leben anhaltenden - Betrachtung des eigenen Handelns in seinen globalen Bezügen anzuregen.

Dass es im Lernbereich Globale Entwicklung auch um die Förderung von Interesse geht, erscheint gemeinhin als selbstverständlich. Dies ist auch im Kontext des wissenschaftlichen Diskurses um Globales Lernen plausibel, wenngleich die Förderung von Interesse hier kaum in expliziter Form als Zieldimension auftaucht. In handlungstheoretischen Konzepten Globalen Lernens kommt die Überzeugung zum Tragen, dass globalen Herausforderungen nur durch das Engagement von Menschen begegnet werden kann (vgl. zu unterschiedlichen Verständnissen Globalen Lernens Asbrand \& Scheunpflug, 2014). Globales Lernen ist in dieser handlungstheoretischen Perspektive ein Konzept, das auf die Entwicklung von Handlungskompetenzen abzielt, auf die Partizipation und das Mitwirken an einer nachhaltigen Gestaltung der Entwicklung der Welt. Dass Interesse hier eine zentrale Funktion zukommt, ist theoretisch plausibel, denn das Konzept des Interesses ist eng verbunden mit dem Konzept der Motivation und kann als zentrales Konstrukt zur Erklärung überdauernder Verhaltensbereitschaften gefasst werden (z.B. Schiefele, 2009).

Aber auch aus Perspektive eines system- und evolutionstheoretischen Verständnisses Globalen Lernens erscheint die Förderung von Interesse als Zielperspektive plausibel. Hier werden die Globalisierung und die Entwicklung zur Weltgesellschaft als mehrdimensionale Komplexitätssteigerung und im Hinblick auf die sich hierdurch ergebenden Lernherausforderungen beschrieben (Scheunpflug, 1996, 2003). Aus dieser Perspektive ist Globales Lernen zentral darauf ausgerichtet, Lernerfahrungen zu ermöglichen im Hinblick auf die Orientierung in einer von einem schnellen sozialen Wandel geprägten Welt, in der Wissen schnell veraltet und das individuelle Nichtwissen stetig zunimmt und in der die Menschheit vor den ungewissen Anforderungen einer kontingenten Zukunft mit komplexen Überlebensfragen konfrontiert ist. Die Erhöhung von Eigenkomplexität angesichts einer sich fortsetzenden Komplexitätssteigerung (Scheunpflug \& 
Schröck, 2000) kommt dabei als Aufgabe lebenslangen Lernens in den Blick. Die Auseinandersetzung mit zentralen Schlüsselproblemen der Gegenwart und Zukunft (Klafki, 1993) ${ }^{1}$ kann nicht als ein sich im schulischen Kontext abschließend vollziehender Lernprozess verstanden werden. Vielmehr wird das Zurechtfinden in einer komplexen Welt immer wieder auf neue und sich fortsetzende Auseinandersetzungs- und Lernprozesse angewiesen sein. Zielsetzung schulischen Unterrichts zu (globalen) Anforderungen der Gegenwart und Zukunft muss es entsprechend sein, nicht nur momentane Auseinandersetzung anzuleiten, sondern auch zu andauernder Beschäftigung jenseits schulischer Leistungsanforderungen anzuregen (Prenzel 1996, S. 1326). Auch aus dieser Perspektive kommt damit die Förderung intrinsischer Motivationslagen und folglich die Förderung von Interesse als relevante Zielperspektive des Unterrichts in den Blick. Aus einer evolutionstheoretischen Perspektive ist dies insbesondere deshalb zentral, weil globale Fragestellungen sich dadurch auszeichnen, dass sie meist nicht im Nahbereich menschlicher Erfahrung liegen und aus diesem Grund oftmals nicht als unmittelbar relevant wahrgenommen werden (Treml, 1998, 1999). Durch schulischen Unterricht eine interessensgeleitete, überdauernde Auseinandersetzung mit solchen Themen zu unterstützten, kann damit bedeuten, einen Beitrag zur (politischen) Mündigkeit Heranwachsender in einer globalisierten Welt zu leisten.

Vor dem Hintergrund der skizzierten Überlegungen erscheint es verwunderlich, dass der Aspekt der Förderung von stabilem Interesse an globalen Fragestellungen im vorwiegend erziehungswissenschaftlichen und auch bildungspolitischen (KMK \& BMZ, 2016) Diskurs um Globales Lernen nur bedingt als eigenständige Zieldimension expliziert wird. Entsprechend nimmt eine explizite und durch empirisch fundierte Theorien aus der Pädagogischen Psychologie angereicherte Auseinandersetzung zur Frage der Interessenförderung bislang wenig Raum ein. Hier setzt der vorliegende Artikel an. Ziel ist es, den Diskurs um Globales Lernen durch die Rezeption von zentralen Theorien der Interessenentwicklung anzureichern und damit sowohl einen Beitrag zur theoretischen Weiterentwicklung des Globalen Lernens zu leisten als auch relevante konzeptionell-didaktische Implikationen aufzuzeigen. Hierzu werden im Folgenden zwei Theorieofferten zunächst in prägnanter Form beschrieben und anschließendals Reflexionsfoliefür bestehendetheoretischewieempirische Auseinandersetzungen im Feld Globalen Lernens fruchtbar gemacht.

\section{Grundzüge der Interessentheorie}

Wenn mit Blick auf Fragen der Unterrichtsgestaltung von der Förderung von Interesse die Rede ist, geht es dabei zunächst um das für den Rahmen des Unterrichts erforderliche situationale Interesse, d.h. um einen aktuellen motivationalen Zustand (Krapp, 1998). Vor dem Hintergrund der skizzierten Überlegungen kommt darüber hinaus die Frage in den Blick, wie individuelle (und damit gerade nicht situationsgebundene) Interessen entstehen und - mit Blick auf die pädagogische Praxis - wie dieser Prozess gefördert werden kann.

In der pädagogischen Psychologie werden zur Interessenentwicklung in theoretischer Perspektive zentral das Person-Gegenstands-Konzept nach Krapp (1992) und die Selbstbestimmungstheorie nach Deci und Ryan (1993) beschrieben (Krapp 2010). Aufgrund ihrer zentralen Stellung im Diskurs wird die
Frage der Interessenförderung im Lernbereich Globale Entwicklung in diesem Artikel unter Bezugnahme auf diese beiden Theorien beleuchtet. Nach dem Person-Gegenstands-Konzept wird Interesse als ein relationales Konstrukt interpretiert, als eine spezifische Person-Gegenstands-Beziehung. Dabei ist der Gegenstand ein subjektiv bestimmter Umweltausschnitt bzw. eine Sinneinheit. Ein persönliches Interesse unterscheidet sich von anderen Person-Gegenstands-Beziehungen vor allem durch eine bedeutungsmäßige Akzentuierung sowie durch mit dem Gegenstand verbundene positive Erlebnisqualitäten. Dem Interessensgegenstand wird demnach erstens eine hohe persönliche Wertigkeit bzw. Relevanz beigemessen, „[d]ie Person [...] fühlt sich davon subjektiv betroffen“ (Krapp, 1992, S. 322). Zweitens ist die Auseinandersetzung mit dem Gegenstand mit einer positiven emotionalen Gesamteinschätzung verbunden, die Person hat positive Erinnerungen an vorherige Auseinandersetzungen mit dem Gegenstand und erwartet, dass hierbei auch zukünftig positive Empfindungen ausgelöst werden (Krapp, 1992). Anknüpfend hieran postuliert Krapp (2010) mit Blick auf die Frage nach der Interessenentwicklung, „dass sich eine Person nur dann mit einem bestimmten Gegenstand dauerhaft und aus innerer Neigung auseinander[setzt], wenn sie ihn auf Basis rationaler Überlegungen als hinreichend bedeutsam einschätzt und wenn sich für sie im Verlauf gegenstandsbezogener Auseinandersetzungen eine insgesamt positive Bilanz emotionaler Erlebensqualitäten ergibt" (S. 317). Für die Entwicklung von Interesse sind demnach sowohl Aspekte kognitiv-rationaler als auch emotionaler Steuerung relevant.

Nach der Selbstbestimmungstheorie von Deci und Ryan (1993) sind für den Bereich emotionaler Steuerung bestimmte Gruppen von Erlebnisqualitäten von besonderer Relevanz. Dieser These liegt die empirisch gestützte Annahme zugrunde, dass die Entstehung von Motivation funktional auf der Befriedigung grundlegender Bedürfnisse basiert. Diese Grundbedürfnisse umfassen das Bedürfnis nach Kompetenzerfahrung (das Bestreben, sich selbst als handlungsfähig zu erfahren), das Bedürfnis nach Autonomieerfahrung (das Bestreben, sich als eigenes Handlungszentrum zu erfahren) sowie das Bedürfnis nach sozialer Eingebundenheit (das Bestreben nach befriedigenden Sozialkontakten) (Krapp, 1998). Demnach hat der Mensch die „angeborene motivationale Tendenz, sich mit anderen Personen in einem sozialen Milieu verbunden zu fühlen, sich mit seinen Fähigkeiten in dieses Milieu wirksam einzubringen und sich dabei persönlich autonom und initiativ zu erfahren" (Prenzel et al., 2000, S. 169). Die Frage, ob und in welchem Maße ein Interesse entwickelt wird und darauf aufbauend eine selbstinitiierte Auseinandersetzung ohne äußere Anreize unterstützt wird, hängt somit zentral mit der Befriedigung dieser drei Grundbedürfnisse zusammen (Krapp, 1998).

\section{Interessenförderung im Lernbereich Globale Entwicklung}

Vor dem Hintergrund der beiden skizzierten Theorieperspektiven benennen Schiefele (2004) sowie Schiefele und Streblow (2006) vier Aspekte der Interessenförderung in schulischem Unterricht: die Förderung von Kompetenzerleben, von Autonomieerleben, von sozialer Eingebundenheit und von subjektiver Bedeutsamkeit. Für den Lernbereich Globale Entwicklung ergeben sich mit Blick auf diese vier Aspekte insbesondere vor dem Hintergrund 
bestehender empirischer Erkenntnisse lernbereichsspezifische Herausforderungen, die im Folgenden erläutert werden.

\section{Förderung von Kompetenzerleben: Den Umgang mit Komplexität schulen}

Nach Schiefele (2004) zielt die Förderung von Kompetenzerleben darauf ab, dass in der Auseinandersetzung mit einem Gegenstand im schulischen Unterricht „das Vertrauen in die eigenen Fähigkeiten [gestärkt wird] " (S. 255), dass sich Lernende in der Beschäftigung mit einem Thema als kompetent wahrnehmen. Für Globales Lernen kommt dabei in den Blick, dass es sich bei den Themen des Lernbereichs um komplexe globale Problemlagen handelt, die nicht mit einfachen, monokausalen Zusammenhängen erklärt werden können und ebenso wenig leicht zu lösen sind. Es stellt sich die Frage, wie in diesem komplexen Kontext Kompetenzerleben gefördert werden kann. Dass es sich hierbei um eine Herausforderung handelt, wird beispielhaft sichtbar an einer empirischen Studie zu Orientierungen von Schülerinnen und Schülern, die im Kontext der Schule Kinderpatenschaften für Kinder im Globalen Süden übernommen haben (Wagener, 2018). Die Studie zeigt auf, dass Jugendliche im Kontext der Kinderpatenschaft eine emotionale Betroffenheit von der Not eines Kindes bzw. der Armut in Ländern des Globalen Südens empfinden und sie sich gleichzeitig als handlungsfähig im Hinblick auf die wahrgenommene Problemlage erfahren: Durch ihre Spenden unterstützen sie ein Kind in einem ärmeren Land. In der Studie wird aber darüber hinaus aufgezeigt, dass die Komplexität globaler sozialer Fragen dabei nicht in den Blick kommt und kein Lernen im Hinblick auf komplexe Hintergründe von sozialen Ungleichheiten in der Welt stattfindet. Dem Handeln in Form von Spenden kommt hier eine Entlastungsfunktion angesichts emotionaler Betroffenheit zu, es geht nicht mit einer weiterführenden Reflexion einher (Wagener, 2018, S. 192). Der Befund unterstützt das empirische Ergebnis Asbrands (2007), dass Jugendliche, die an der entwicklungspolitischen Praxis des Helfens teilhaben, ein asymmetrisches Nord-Süd-Verständnis und damit ein „nicht hinreichend komplexe[s] Weltbild“ (S. 14) konstruieren. In (system)theoretischer Perspektive ist dies anschlussfähig an die Argumentation von Luhmann (1975, S. 145) und Treml (1998, S. 11), dass konkrete Hilfe eine im Nahbereich funktionale Form des Miteinanders ist, die im weltgesellschaftlichen Kontext unterkomplex und für eine dahingehende Orientierung daher dysfunktional ist. Offenbar wird hier also, dass Lerngelegenheiten des Globalen Lernens der Komplexität weltgesellschaftlicher Realität stärker entsprechen müssen als es beim Beispiel des Lernsettings Kinderpatenschaft der Fall ist. An diesem beispielhaften empirischen Befund wird in theoretischer Perspektive der schmale Grat sichtbar zwischen einerseits der Ermöglichung von Kompetenzerleben (z. B. in Form eines Erfahrens von Handlungsfähigkeit durch Spenden) und andererseits der Ermöglichung eines Lernens im Hinblick auf die Komplexität globaler Problemlagen (was auch die Erkenntnis umfasst, dass die eigenen Möglichkeiten, unmittelbare Veränderungen herbeizuführen, beschränkt sind). Die Frage ist daher, wie einerseits die begrenzten Handlungs- und Einflussmöglichkeiten als solche betrachtet werden können und andererseits Handlungs- und Selbstwirksamkeitserfahrungen ermöglicht werden können, die möglichen Ohnmachts- und Überforderungsgefühlen entgegenwirken. Asbrand (2009) hat in ihrer Studie zu Jugendlichen, die sich in unterschiedlichen Settings mit globalen Entwicklungsfragen beschäftigt haben, herausgearbeitet, dass es von den Strategien der Komplexitätsreduktion abhängt, „inwiefern sich Jugendliche als handlungsfähig wahrnehmen“" (S. 238). Für Unterricht im Lernbereich Globale Entwicklung ergibt sich demnach die Herausforderung, Lernarrangements so zu gestalten, dass Möglichkeiten der Komplexitätsreduktion kennengelernt werden können und gleichzeitig zur Erschließung von Komplexität angeregt wird. Als besondere Herausforderung ergibt sich hierbei, dass für den Lernbereich Globale Entwicklung bislang keine empirisch basierten Kompetenzstrukturmodelle vorliegen (vgl. Asbrand, 2014), die die Identifikation und Entwicklung von Lernkontexten und Aufgabenformaten ermöglichen würden, die unterschiedliche Kompetenzstände ansprechen und eine Weiterentwicklung anregen. Angesichts des weiterhin bestehenden Theorie- und Empiriedefizits im Globalen Lernen ergibt sich in didaktischer Perspektive damit ein Spannungsfeld zwischen unsachgemäßer Komplexitätsreduktion und potenzieller Überforderung. Während ersteres die spezifische Anforderung des Lernbereichs, d. h. die kognitive Erschließung abstrakter Lerngegenstände, untergräbt, steht letzteres der Ermöglichung von Kompetenzerleben und damit der Ausbildung und Entfaltung von Interesse potenziell entgegen. Für das didaktische Vorgehen erscheint es vor diesem Hintergrund sinnvoll, Auswirkungen von eigenen Handlungen im Kleinen sichtbar zu machen und als wertvoll zu erkennen und gleichzeitig zur Reflexion der Grenzen des eigenen Beitrags anzuregen (vgl. Wagener, 2014).

\section{Förderung von Autonomieerleben: (Weltgesellschaftliche) Partizipation ermöglichen}

Beim Aspekt der Förderung von Autonomieerleben geht es nach Schiefele (2004) zentral darum, dass sich Schülerinnen und Schüler in der unterrichtlichen Auseinandersetzung mit Inhalten als selbstbestimmt handelnd wahrnehmen. Für den Bereich Globalen Lernens ist an dieser Stelle die Frage des Umgangs mit der impliziten Normativität, der Ausrichtung an den Leitbildern Nachhaltigkeit und Gerechtigkeit, relevant. An dieser Frage ergab sich der sogenannte Paradigmenstreit zwischen handlungs- und systemtheoretischen Verständnissen Globalen Lernens (vgl. Asbrand \& Scheunpflug, 2014). Aus der Perspektive handlungstheoretischer Entwürfe werden normative Ziele sowie Inhalte Globalen Lernens formuliert, die mit darauf ausgerichteten Bildungsangeboten erreicht werden sollen. Gegen eine derartige, sogenannte normative "Postulativpädagogik" (Scheunpflug \& Seitz, 1993, S. 70) wenden sich system- und evolutionstheoretische Entwürfe, die weniger von Ideen zur gezielten Veränderung der gesellschaftlichen Verhältnisse geprägt sind und ihren Ausgangspunkt bildungstheoretisch vielmehr bei den Lernenden als sich in der Globalisierung befindenden Bildungssubjekten sehen. Aus empirischer Sicht legen verschiedene Studien (Asbrand, 2009; Applis, 2012; Holfelder, 2018; Wagener, 2018; Kater-Wettstädt, 2015) nahe, dass normativ-moralische Handlungsaufforderungen im Unterricht mit abweisendem Verhalten von Schülerinnen und Schülern einhergehen. So zeigten sich in der oben genannten Studie von Asbrand (2009) bei Jugendlichen, die sich im schulischen Unterricht mit dem Themenfeld Globalisierung auseinandergesetzt hatten, unterschiedliche Formen der Distanzierung von moralischen Ansprüchen, z. B. über Entschuldigungsstrategien. Kater-Wettstädt (2015) interpretiert solche Dis- 
tanzierungen als Reaktion auf „moralische Überwältigung“ (S. 263). Applis (2012) rekonstruiert bei Schülerinnen und Schülern vom Gymnasium mit Blick auf den Geographieunterricht unter anderem einen intellektualisierenden Weltzugang (S. 266ff.), bei dem Jugendliche nach einer differenzierten Auseinandersetzung streben und „sich gegen jede dichotomisierende moralische Perspektive [stellen]“ (S. 271). In der Studie zu Jugendlichen in Kinderpatenschaften zeigt sich eine Distanzierung von der Auseinandersetzung mit Entwicklungsfragen als Reaktion auf mangelnde Authentizität des Engagementsettings Patenschaft: Eine solche Abweisung ergibt sich offenbar gerade dann, wenn die Idee der Übernahme einer Patenschaft (z.B. durch Lehrkräfte) mit einem normativen Impetus an die Jugendlichen herangetragen und moralisch eingefordert wurde (Wagener, 2018, S. 186). Die empirischen Befunde geben damit Hinweise darauf, dass Globales Lernen im Sinne einer Förderung von Autonomieerleben Möglichkeiten echter, freiwilliger Partizipation bedarf. Für eine Förderung von Interesse (und eine Verhinderung von Abwendung) bedarf es ausreichender Handlungsspielräume und Wahlfreiheiten in der Auseinandersetzung mit globalen Themen. Dies kann für Globales Lernen im schulischen und einem damit gerade nicht freiwilligen Kontext eine Herausforderung darstellen, insbesondere wenn seitens der Lehrkraft Aktions- und Engagementformate in die Beschäftigung mit globalen Themen integriert werden (hierzu Asbrand \& Kater-Wettstädt, 2014).

\section{Förderung sozialer Eingebundenheit:}

\section{Zur Reflexion von Wertmaßstäben anregen}

Aus motivations- und interessenstheoretischer Sicht wird die Befriedigung des Bedürfnisses nach sozialer Eingebundenheit als eine zentrale Voraussetzung der Bereitschaft zu einer andauernden Auseinandersetzung mit einem Themengebiet verstanden (vgl. Krapp, 1998). Eine Herausforderung ergibt sich in dieser Perspektive insbesondere im Kontext eines handlungstheoretisch verstandenen Globalen Lernens, welches an normativen Lernzielen im Sinne von gewünschten Einstellungsänderungen orientiert ist. Wie es vor dem Hintergrund des Bedürfnisses nach sozialer Eingebundenheit plausibel erscheint, hat Holfelder (2018) empirisch aufgezeigt, dass Jugendliche in der Auseinandersetzung mit nachhaltigkeitsbezogenen Themen an Konformität orientiert sind und entsprechend dazu tendieren, Handlungsalternativen zu wählen, die denen ihrer Peergroup entsprechen. Eine im Unterricht zu globalen Themen stattfindende normative Einforderung bestimmter Einstellungen und Verhaltensweisen läuft dieser Orientierung tendenziell entgegen. Dies zeigt sich an verschiedenen Stellen des empirischen Materials der Studie und wird beispielhaft deutlich, wenn die Jugendlichen in den für die Studie geführten Gruppendiskussionen auf Fragen des Kleidungskaufs zu sprechen kommen. Die im Nachhaltigkeitsdiskurs normativ gesetzte Perspektive, aufgrund von ökologischen und sozialen Problemstellungen beispielsweise weniger Kleidung zu kaufen und dabei trotz finanziellen Mehraufwandes auf nachhaltig agierende Unternehmen zurückzugreifen, steht in Konflikt mit dem von den Jugendlichen allgemein und insbesondere in ihrer Peergroup als gängig erfahrenen Konsumverhalten und wird entsprechend nicht als Option für die eigene Handlungspraxis erachtet (Holfelder, 2018, S. 291ff.). Obwohl Jugendlichen in vielen Studien ein hohes Bewusstsein für Fragen globaler Entwicklung und Nachhaltigkeit attestiert wird (u.a. Michelsen, et al. 2015; Bertelsmann
Stiftung, 2009; Shell, 2010), zeigt sich empirisch gleichzeitig auch ein Konkurrenzverhältnis zwischen das eigene Leben unmittelbar betreffenden und nachhaltigkeitsrelevanten Werten (Leitner, 2011). Dass z.B. nachhaltigkeitsorientiertes Konsumverhalten für viele Jugendliche dem persönlichen Lebensstil entgegenläuft, ist ein Ergebnis einer Befragung von Jugendlichen der 9. und 10. Klasse in einer Studie von Zubke (2005). Mit Blick auf die Förderung von Interesse weisen auch diese empirischen Ergebnisse auf eine weitere Problematik eines auf Einstellungs- und Verhaltensänderung abzielenden Globalen Lernens hin. In didaktischer Perspektive erscheint es vor diesem Hintergrund sinnvoll, in der Schule nicht normativ eng die eine Moral zu vermitteln und entsprechend erwünschte Verhaltensänderungen als Ziel von Unterricht zu setzen, sondern vielmehr zur Reflexion unterschiedlicher Kriterien und Maßstäbe anzuregen (hierzu auch Kater-Wettstädt, 2015, S. 263ff.).

\section{Förderung subjektiver Bedeutsamkeit: Die Relevanz globaler Themen erhöhen}

Beim letzten Aspekt der Interessenförderung geht es im Gegensatz zu den drei bislang diskutierten Bereichen nicht darum, im Lernprozess didaktisch auf die Befriedigung der drei grundlegenden Bedürfnisse (Deci \& Ryan, 1993) zu achten, sondern vielmehr darum, „den subjektiven Wert von Lerngegenständen [...] direkt zu erhöhen“ (Schiefele, 2004, S. 257). Zunächst erscheint es plausibel, dass die Themenfelder Globalen Lernens, die oftmals Zukunftsfragen der Menschheit betreffen, im Vergleich zu anderen, wenig lebensweltbezogenen Gegenständen eine größere Bedeutsamkeit für Schülerinnen und Schüler enthalten. Gleichzeitig nehmen, wie eingangs bereits angemerkt, die persönlichen Relevanzen aus evolutionstheoretischer Sicht mit der räumlichen und/oder zeitlichen Entfernung ab. Mit dieser Perspektive wird verständlich, warum Menschen trotz des Wissens um langfristige Auswirkungen ressourcenintensiv leben: Entgegen des Prinzips der Nachhaltigkeit, welches zukünftigen und derzeitig lebenden Generationen das gleiche Recht auf Befriedigung ihrer Bedürfnisse zuspricht, werden die eigenen, aktuellen Bedürfnisse als relevanter wahrgenommen und für Verhaltensentscheidungen priorisiert.

In der pädagogischen Psychologie wird als didaktisches Mittel zur Förderung subjektiver Bedeutsamkeit die Verschränkung mit anderen, bereits bestehenden Interessensgegenständen in Form einer Kontextualisierung genannt (Schiefele, 2004). Der Lerngegenstand wird hier im Anschluss an den Schülerinnen und Schülern bekannte Alltagskontexte oder im Sinne (vermeintlich) bestehender Interessensbereiche situiert. Eine Kontextualisierung im Alltagskontext bzw. im Nahbereich individueller Erfahrung ist mit Blick auf Globales Lernen gerade deshalb möglich, weil viele globale Themen durch Glokalität gekennzeichnet sind. Mit dem Begriff Glokalisierung (Robertson, 1998) wird die Verwobenheit von lokalen und globalen Prozessen beschrieben und darauf verwiesen, dass beispielsweise im Kontext der Textilindustrie die lokale Erscheinung eines Bekleidungsgeschäftes oder schon das Tragen eines Kleidungsstückes am eigenen Körper durchdrungen ist mit der globalen Dimension einer sich über den Globus erstreckenden Herstellung von Kleidung. Zum einen ist die Entwicklung eines Verständnisses für diese glokalen Verflechtungen eine Zielperspektive Globalen Lernens und zum anderen besteht im Aufzeigen lokaler Relevanzen globaler Prozesse eine Möglichkeit 
der Förderung von subjektiver Bedeutsamkeit des Gegenstandes und damit Interesse.

Im Hinblick auf die Verknüpfung des Lerngegenstandes mit bestehenden Interessensgebieten ist es eine besondere Chance des Lernbereiches, dass Themenbereiche des Lernfeldes Globale Entwicklung sich in unterschiedlichen Fächern und damit auch fächerübergreifend behandeln lassen (KMK \& BMZ, 2016). Beispielweise das Thema Energie kann in technischer Perspektive im Hinblick auf Herausforderungen der Energieversorgung in ländlichen Regionen sowie in sozialer Hinsicht mit Blick auf die sich durch eine mangelhafte Energieversorgung ergebenden eingeschränkten Lebens- und Teilhabeperspektiven thematisiert werden. Es besteht also die Möglichkeit, unterschiedlichen Schülerinnen und Schülern einen individuell interessanten Zugang zu globalen Fragestellungen zu ermöglichen. Gleichzeitig kommt die Frage danach auf, was in verschiedenen Kontextualisierungen subjektiv zum Gegenstand der Auseinandersetzung bzw. zum Interessensgegenstand wird. So ist aus Forschungen zu situiertem Lernen im Mathematikunterricht bekannt, dass Kontextualisierungen von den abstrakten, zu erwerbenden Inhalten ablenken können (Stark, Gruber \& Mandl, 1998; Moschner \& Schiefele, 2000). Für das Globale Lernen bedeutet dies, dass Situierungen in einer Weise erfolgen müssen, dass diese dem Lernziel einer abstrakten Erschließung komplexer Fragen nicht entgegenstehen. Als Herausforderungsichtbarwird dieserneutanderStudiezuLernerfahrungen von Jugendlichen in Kinderpatenschaften (vgl. Wagener, 2018). Die Übernahme einer Kinderpatenschaft im Kontext von Schule kann als eine didaktisch intendierte Situierung des Lernens begriffen werden: Das Thema der großen sozialen Disparitäten in der Welt wird in der Patenschaft in Form einer persönlichen Unterstützungsbeziehung zwischen zwei konkreten Menschen aufgegriffen. Die Ergebnisse der Studie zeigen jedoch, dass die Auseinandersetzung im Kontext dieser didaktischen Figur auf den Einzelfall konzentriert bleiben kann und die globale Dimension der Patenschaft nur bedingt in den Blick kommt.

Nach Schiefele (2004) ist ein weiteres Mittel zur Erhöhung der subjektiven Bedeutsamkeit die Erhöhung des emotionalen Gehalts des Lerngegenstandes. In der Praxis Globalen Lernens findet sich eine solche Emotionalisierung beispielsweise in der didaktischen Nutzung von Katastrophen- und Schreckensszenarios im Hinblick auf den Klimawandel sowie in der geplanten Weckung von Mitleidsempfindungen. Eine solche Emotionsdidaktik ist mit Blick auf die Förderung von Interesse zunächst plausibel und geht zudem - wie Erfahrungen aus Marketing und Spendenakquise zeigen - mit bestimmten Verhaltenbereitschaften wie Spenden einher. Gleichzeitig verweisen empirische Ergebnisse darauf, dass im Kontext starker Emotionalisierungen Reflexionsbzw. Verstehensprozesse zurückbleiben können. Sowohl die Studien von Tallon (2012) als auch von Wagener (2018) weisen auf einen Zusammenhang von emotionaler Betroffenheit, z.B. in der Konfrontation mit schwierigen Lebenssituationen anderer Menschen, und dem Bedürfnis nach einer Selbstvergewisserung durch Handeln hin. Die Wahl von leicht zugänglichen Handlungsoptionen wie dem Spenden angesichts der medial sichtbaren Not eines Kindes in einem Land des Globalen Südens können dabei als (implizite) „Abkürzungsstrategien im Hinblick auf eine Auseinandersetzung mit komplexen Hintergründen sozialer Disparitäten in der Welt interpretiert werden" (Wagener, 2018, S. 194). Damit wird ein für das Globale Lernen spezifisches Spannungsfeld bezüglich des Verhältnisses von Kognition und Affektion sichtbar. So erscheint Betroffenheit auf der einen Seite sowohl aus einer evolutionstheoretischen als auch einer interessentheoretischen Perspektive als wichtiges Moment der Auseinandersetzung mit globalen Problemlagen und auch empirische Studien weisen auf diese Bedeutung von Betroffenheit für Globales Lernen hin (z. B. Brown, 2015). Auf der anderen Seite kann gerade Betroffenheit mit dem Bedürfnis schneller Abhilfe einhergehen und damit eine weiterführende kognitive Erschließung des Problemkontextes beeinträchtigen (Wagener, 2018, S. 194). Auch hinsichtlich dieses Aspektes der Förderung von Interesse bedarf es entsprechend einer reflexiven Didaktik im Umgang mit dem Spannungsfeld zwischen der Annäherung an Interessen und Bedürfnisse der Lernenden und der Orientierung an der Zielperspektive eines Lernens im Hinblick auf die Komplexität abstrakter globaler Fragen. Es erscheint sinnvoll, dass hierbei die Anleitung zur Reflexion der sich in der Auseinandersetzung mit globalen Problemlagen ergebenden Emotionen eine bedeutende Rolle spielt.

\section{Fazit}

Die Förderung von Interesse ist aus verschiedenen im Feld diskutierten Theorieperspektiven ein wichtiger, aber im Diskurs bislang wenig explizierter und theoretisch reflektierter Bestandteil des Unterrichts zu globalen Themen. Vor diesem Hintergrund ist es Ziel dieses Artikels, einen Beitrag zum Diskurs um Globales Lernen aus interessentheoretischer Perspektive zu leisten und darüber hinaus konzeptionell-didaktische Implikationen aufzuzeigen.

In theoretischer Perspektive wurden Herausforderungen einer Interessenförderung im Globalen Lernen sichtbar. Erstens die evolutionstheoretische Argumentation, dass globale Fragestellungen oftmals nicht im Nahbereich menschlicher Erfahrung liegen und daher als nicht unmittelbar relevant eingeordnet werden (Treml, 1998, 1999). Zweitens ergibt sich angesichts der Komplexität globaler Fragestellungen ein Spannungsfeld zwischen einer das individuelle Kompetenzerleben fördernden Komplexitätsreduktion und einer auf die Erschließung von Komplexität ausgerichteten Auseinandersetzung mit globalen Themen. Drittens verweist die interessenstheoretische Diskussion empirischer Befunde auf die Herausforderung im Umgang mit der Normativität des Lernbereichs. Hier wird ein Zusammenhang zwischen einer Setzung von normativen Zielsetzungen im Sinne von Einstellungsund Verhaltensänderungen und der Beförderung von Abwendungstendenzen sichtbar. Eine Rolle spielt dabei insbesondere die offenbar teils schwierige Vereinbarkeit von nachhaltigkeitsorientierten und dem jugendlichen Lebensstil entsprechenden Verhaltensweisen.

Daran anschließend wurde in konzeptionell-didaktischer Hinsicht aufgezeigt, dass Lerngelegenheiten im Sinne der Interessenförderung zum Erkennen und Wertschätzen eigener Handlungsperspektiven anregen sollten, aber angesichts komplexer Wirkungszusammenhänge gleichzeitig zur Erkenntnis der Begrenztheit des eigenen Wirkens beitragen müssen. Zudem liegt es nahe, Lernangebote zu gestalten, die zu einer Reflexion in globaler Dimension anleiten, aber Positionen nicht normativ einfordern. Dazu gehört auch die Wahrung von Gestaltungsspielräumen auf Seite der Lernenden. Chancen der Interessenförderung bestehen insbesondere angesichts der thematischen Breite des Lernbereiches, die vielfältige Zugänge zur Auseinandersetzung mit globalen Themen erlaubt, sowie der glokalen Dimension der Themen, 
die die Bezugnahme auf den Nah- bzw. Erfahrungsbereich der Schülerinnen und Schüler ermöglicht. Wenngleich eine Erhöhung des emotionalen Gehaltes des Lerngegenstandes für die Entwicklung von Interesse förderlich ist, gilt es im Globalen Lernen achtsam mit Gestaltungselementen umzugehen, die auf die Erweckung von Emotionen ausgerichtet sind.

Weiterführend und an dieser Stelle ungeklärt stellt sich abschließend die Frage, auf welche Gegenstände sich das im Unterricht zum Themenfeld globale Entwicklung zu fördernde Interesse eigentlich beziehen soll. Geht es um die Förderung einer übergreifenden Interessensperspektive des Globalen - z.B. als Interesse an Fragen von Gerechtigkeit und Nachhaltigkeit - oder um Interesse bezüglich der für die unterrichtliche Behandlung beispielhaft gewählten Themen wie z.B. dem Klimawandel oder der Kakaoproduktion? Nach Krapp (2010) ist ein Interessensgegenstand ein individuell konstruierter Umweltausschnitt, sodass der tatsächliche Interessensaufbau letztlich von den individuellen Konstruktionen der Lernenden abhängt und damit wie jedes Lernen unverfügbar ist. Offen bleibt die normative Frage nach der interessensbezogenen Zielsetzung des Unterrichts zu globalen Themen. An dieser Stelle empfiehlt sich ein Weiterdenken in bildungstheoretischer Perspektive.

\section{Anmerkung}

1 Schlüsselprobleme werden hier verstanden als unabgeschlossener Kanon von globalen Gegenwarts- und Zukunftsfragen, der aktuell vielleicht am treffendsten durch die globalen Nachhaltigkeitsziele der Vereinten Nationen umrissen werden kann.

\section{Literatur}

Applis, S. (2012). Wertorientierter Geographieunterricht im Kontext Globales Lernen. Theoretische Fundierung und empirische Untersuchung mit Hilfe der dokumentarischen Methode. Weingarten: Hochschulverband für Geographie und ihre Didaktik.

Asbrand, B. (2007). Partnerschaft - Eine Lerngelegenheit? Zeitschrift für Internationale Bildungsforschung und Entwicklungspädagogik, 30(3), 8-14.

Asbrand, B. (2009). Wissen und Handeln in der Weltgesellschaft. Eine qualitativ-rekonstruktive Studie zum Globalen Lernen in der Schule und in der außerschulischen Jugendarbeit. Münster: Waxmann.

Asbrand, B. \& Kater-Wettstädt, L. (2014): Handeln in der Weltgesellschaft. Zum Umgang mit Handlungsaufforderungen im Unterricht zu Themen des Lernbereichs Globale Entwicklung. Zeitschrift für Internationale Bildungsforschung und Entwicklungspädagogik, 37(1), 4-12.

Asbrand, B. \& Scheunpflug, A. (2014). Globales Lernen. In W. Sander (Hrsg.), Handbuch politische Bildung (S. 401-412). Bonn: BpB.

Bertelsmann Stiftung. (2009). Jugend und die Zukunft der Welt: Ergebnisse einer repräsentativen Umfrage in Deutschland und Österreich „Jugend und Nachhaltigkeit". Veröffentlicht unter: $\quad$ https://www.bertelsmann-stiftung.de/fileadmin/files/BSt/Presse/im ported/downloads/xcms_bst_dms_29232_29233_2.pdf [20.01.2019]

Brown, K. (2015). Responding to global poverty: young people in England learning about development. Dissertation, University College London. London.

Deci, E. \& Ryan, R. (1993). Die Selbstbestimmungstheorie der Motivation und ihre Bedeutung für die Pädagogik. Zeitschrift für Pädagogik, 39(2), 223-238.

Holfelder, A.-K. (2018). Orientierungen von Jugendlichen zu Nachhaltigkeitsthemen. Zur didaktischen Bedeutung von implizitem Wissen im Kontext BNE. Wiesbaden: Springer VS. doi.org/10.1007/978-3-658-18681-4

Kater-Wettstädt, L. (2015). Unterricht im Lernbereich globale Entwicklung. Der Kompetenzerwerb und seine Bedingungen. Münster: Waxmann.

Klafki, W. (1993). Allgemeinbildung heute. Grundzüge internationaler Erziehung. Pädagogisches Forum, 6(1), 21-28.

KMK \& BMZ (Hrsg.) (2016). Orientierungsrabmen für den Lernbereich Globale Entwicklung im Rahmen einer Bildung furr nachhaltige Entwicklung. Bonn: Cornelsen.

Krapp (1992). Das Interessenkonstrukt: Bestimmungsmerkmale der Interessenhandlung und des individuellen Interesses aus der Sicht einer Person-Gegenstands-Konzeption. In
A. Krapp \& M. Prenzel (Hrsg.), Interesse, Lernen, Leistung. Nenere Ansätze der pädagogisch-psychologischen Interessenforschung (S. 297-329). Münster: Aschendorff.

Krapp, A. (1998). Entwicklung und Förderung von Interessen im Unterricht. Psychologie in Erziehung und Unterricht, 44, 185-201.

Krapp, A. (2010). Interesse. In D. H. Rost (Hrsg.), Handwörterbuch Pädagogische Psychologie (4. Aufl., S. 311-323). Weinheim: Beltz.

Leitner, M. (2011). Jugendliche Lebensstile und Nachhaltigkeit. Österreichische Zeitschrift fuir Soziologie, 36(2), 109-119. doi.org/10.1007/s11614-011-0036-9

Luhmann, N. (1975). Formen des Helfens im Wandel gesellschaftlicher Bedingungen. In N. Luhmann (Hrsg.), Soziologische Aufklärung 2. Aufsätze zur Theorie der Gesellschaft (S. 134-149). Opladen: Westdeutscher Verlag. doi.org/10.1007/978-3-663-12374-3_7

Michelsen, G., Grunenberg, H., Mader, C., \& Barth, M. (2015). Greenpeace Nachhaltigkeitsbarometer 2015 - Nachhaltigkeit bewegt die jüngere Generation. Veröffentlicht unter https://www.greenpeace.de/sites/www.greenpeace.de/files/publications/nachhal tigkeitsbarometer-2015-zusammenfassung-greenpeace-20160113_0.pdf [20.01.2019]

Moschner B. \& Schiefele U. (2000). Motivationsförderung im Unterricht. In M.K.W. Schweer (Hrsg.), Lebrer-Schüler-Interaktion. Pädagogisch-psychologische Aspekte des Lehrens und Lernens in der Schule (S. 177-193). Wiesbaden: Springer VS. doi.org/10.1007/9783-322-97477-8_9

Prenzel, M. (1996). Mit Interesse in das dritte Jahrtausend! Pädagogische Überlegungen. In N. Seibert, \& H.J. Serve (Hrsg.), Bildung und Erziehung an der Schwelle zum dritten Jahrtausend (S. 1314-1339). Marquartstein: PimS.

Prenzel, M., Drechsel, B., Kliewe, A., Kramer, K. \& Röber, N. (2000). Lernmotivation in der Aus- und Weiterbildung: Merkmale und Bedingungen. In C. Harteis, H. Heid \& S. Kraft (Hrsg.), Kompendium Weiterbildung Aspekte und Perspektiven betrieblicher Personal- und Organisationsentwicklung (S. 163-17). Wiesbaden: Springer VS. doi. org/10.1007/978-3-322-97460-0_14

Scheunpflug, A. (1996). Die Entwicklung zur globalen Weltgesellschaft als Herausforderung für das menschliche Lernen. Zeitschrift fuir Internationale Bildungsforschung und Entwicklungspädagogik, 19(1), 9-14.

Scheunpflug, A. (2003). Globalisierung als Bildungsherausforderung. In J. Beillerot \& C. Wulf (Hrsg.), Erziehungswissenschaftliche Zeitdiagnosen. Deutschland und Frankreich (S. 262-278). Münster: Waxmann.

Scheunpflug, A. \& Schröck, N. (2000). Globales Lernen. Einführung in eine pädagogische Konzeption zur entwicklungsbezogenen Bildung. Hrsg. von Brot für die Welt. Stuttgart.

Scheunpflug, A. \& Seitz, K. (1993). Entwicklungspädagogik in der Krise? Versuch einer Zwischenbilanz. In A. Scheunpflug \& K. Seitz (Hrsg.), Selbstorganisation und Chaos: Entwicklungspolitik und Entwicklungspädagogik in neuer Sicht (S. 57-75). Tübingen: Schöppe und Schwarzenbart.

Schiefele, U. (2009). Motivation. In E. Wild \& J. Möller (Hrsg.), Pädagogische Psychologie (S. 151-176) Heidelberg: Springer Medizin Verlag. doi.org/10.1007/978-3-540-885733_7

SHELL-Studie: Albert, M. (2010). Jugend 2010: Eine pragmatische Generation behauptet sich. Frankfurt a.M.: Fischer-Taschenbuch-Verlag.

Stark, R., Gruber, H. \& Mandl, H. (1998). Motivationale und kognitive Passungsprobleme beim komplexen situierten Lernen. Psychologie in Erziehung und Unterricht, 44, 202-215.

Tallon, R. (2012). Emotion and Agency within NGO Development Education: what is at work and what is at stake in the classroom? International Journal of Development Education and Global Learning, 4(2), S. 5-22. doi.org/10.18546/IJDEGL.04.2.02

Treml, A. K. (1998). Globales Lernen oder: Die Überforderung der Pädagogik durch die Weltgesellschaft. Zeitschrift für Internationale Bildungsforschung und Entwicklungspädagogik, 21(3), 8-12.

Treml, A. K. (1999). Die Erziehung zum Weltbürger. In D. Neumann, A. Schöppe \& A. K. Treml (Hrsg.), Die Natur der Moral. Evolutionäre Ethik und Erziehung (S. 177-194). Stuttgart: Hirzel.

Wagener, M. (2014). Entwicklungspolitische Bildung durch Kinderpatenschaften? Praxis Gemeindepädagogik, 67(1), 43-45.

Wagener, M. (2018). Globale Sozialität als Lernherausforderung. Eine rekonstruktive Studie $z u$ Orientierungen von Jugendlichen in Kinderpatenschaften. Wiesbaden: Springer VS. doi. org/10.1007/978-3-658-18822-1

Zubke, G. (2005). Umweltbildung im Kontext jugendtypischer Lebensstile - Empirische Untersuchungen zur Bedeutung außerschulischer Faktoren für das Umwelthandeln. In M. Schrenk (Hrsg.), Schriftenreihe Bildung für nachhaltige Entwicklung: Vol. 1. Bildung für nachhaltige Entwicklung. Ergebnisse empirischer Untersuchungen (S. 131-142). Hamburg: Kovač.

\section{Dr. Marina Wagener}

ist wissenschaftlicher Mitarbeiterin am Lehrstuhl für Allgemeine Pädagogik am Institut für Erziehungswissenschaft an der Otto-Friedrich-Universität Bamberg. Ihre Arbeits- und Forschungsschwerpunkte liegen im Bereich Globales Lernen und Bildung für nachhaltige Entwicklung. 